\title{
UNITARY SUBGROUP OF INTEGRAL GROUP RINGS
}

\author{
A.A. Bovdi and S.K. SehgaL
}

Abstract

Lct $A$ be a finite abelian group and $G=A \rtimes\langle b\rangle, b^{2}=1, a^{b}=$ $a^{-1}, \forall a \in A$. We find generators up to finite index of the unitary subgroup of $\mathbb{Z} G$. In fact, the generators are the bicyclic units. For an arbitrary group $G$, let $B_{2}(\mathbb{Z} G)$ denote the group generated by the bicycilic units. We classify groups $G$ such that $B_{2}(\mathbb{Z} G)$ is unitary.

Let $\mathbb{Z} G$ be the integral group ring of an arbitrary group $G$ and let $f: G \rightarrow U(\mathbb{Z})=\{ \pm 1\}$ be an orientation homomorphism. For each $x=\sum_{g \in C^{\prime}} \alpha_{g} g$, we put $x^{f}=\sum \alpha_{g} f(g) g^{-1}$. In particular, if $f$ is trivial, $x^{f}$ coincides with the standard $x^{*}$. Let $U(\mathbb{Z} G)$ be the group of units of $\mathbb{Z} G$. Then $u \in U(\mathbb{Z} G)$ is called $f$-tunitary if $u^{-1}=u^{f}$ or $u^{-1}=-u^{f}$. All $f$-unitary elements of $U(\mathbb{Z} G)$ form a subgroup $U_{f}(\mathbb{Z} G)$ containing $G \times U(\mathbb{Z})$. We refer to $U_{f}(\mathbb{Z} G)$ as the $f$-unitary subgroup of $U(\mathbb{Z} G)$. Interest in the group $U_{f}(\mathbb{Z} G)$ arose in algebraic topology and unitary $K$-theory [4].

We are interested in the constructive description of $U_{f}(\mathbb{Z} G)$. If $G$ is finite cyclic, then Bovdi [ 1 ] gavc a linearly independent set of generators for a torsion free subgroup of finite index in $U_{f}(\mathbb{Z} G)$. This was extended to finite abelian groups by Hoechsmann-Sehgal in [3]. We give generators up to finite index of $U_{f}(\mathbb{Z} G)$ if $G$ is a finite dihedral group. In fact, the generators consist of the bicyclic units. The subgroup $B_{2}(\mathbb{Z} G)$ of $U(\mathbb{Z} G)$ generated by all the bicyclic units of $\mathbb{Z} G$ plays an important role in the study of $U(\mathbb{Z} G)$ (see [5], [6]). In Theorem 2, we characterize groups $G$ for which $B_{2}(\mathbb{Z} G)$ is unitary. 


\section{2. $U_{f}(\mathbb{Z} G)$ for dihedral groups}

First, we recall some definitions. For an element $a \in G$ of finite order $n$ write $\bar{a}=1+a+\cdots+a^{n-1}$. Denote by $t(G)$ the set of all torsion elements of $G$. If $a, b \in G, o(a)<\infty$, then

$$
u_{a, b}=1+(1-a) b \bar{a}
$$

has an inverse $u_{a, b}^{-1}=1-(1-a) b \bar{a}$. Moreover, $u_{a, b}=1$ if and only if $b$ normalizes $\langle a\rangle$. The elements $u_{a, b}, a, b \in G$ are called bicyclic units of $\mathbb{Z} G$ and the group generated by them is denoted by $B_{2}(\mathbb{Z} G)$. We recall [5] that by $B_{1}(Z G)$ is understood the group generated by the Bass cyclic units of $\mathbb{Z} G$. It is known [5] that if $G$ is a finite dihedral group and $Z$ is the centre of $U(\mathbb{Z} G)$ then $\left\langle Z, B_{2}(\mathbb{Z} G)\right\rangle$ (equivalently, $\left\langle B_{1}(\mathbb{Z} G), B_{2}(\mathbb{Z} G)\right\rangle$ ) is of finite index in $U(\mathbb{Z} G)$. We prove

Theorem 1. Let $G$ be the dihedral group

$$
D_{2 n}=\left\langle a^{n}=1=b^{2} \mid a^{b}=a^{-1}\right\rangle .
$$

Suppose $f$ is an orientation homomorphism of $G$ with kernel $\langle a\rangle$. Then the index $\left(U_{f}(\mathbb{Z} G): B_{2}(\mathbb{Z} G)\right)$ is finite.

We need the

Proposition. Let $G$ be a group containing a subgroup $A$ of index 2 and an element $b$ such that $G=\langle A, b\rangle$ and $b^{-1} a b=a^{-1}$ for all $a \in A$. Suppose that $A^{2} \neq 1$. If $f$ is an orientation homomorphism of $G$ with kernel $A$, then

1) the centre of $U_{f}(\mathbb{Z} G)$ coincides with $t_{2}(A) \times\langle-1\rangle$, where

$$
t_{2}(A)=\left\{a \in t(A): a^{2}=1\right\} ;
$$

2) the centre of $U(\mathbb{Z} G)$ is the direct product of $t_{2}(A) \times\langle-1\rangle$ and a torsion free abelian group $T$ such that $U(\mathbb{Z} A)=\langle-I\rangle \times A \times T$ and $x=x^{*}$ for all $x \in T$.

Proof: Let $x=x_{1}+x_{2} b, \quad x_{i} \in \mathbb{Z} A$ be a central unit in $\mathbb{Z} G$. Since $G$ is a subgroup of $U(\mathbb{Z} G)$,

$$
x=b^{-1} x b=x_{1}^{*}+x_{2}^{*} b \quad \text { and } \quad x=a^{-1} x a=x_{1}+a^{-2} x_{2} b
$$

for all $x \in A$. Then $x_{i}=x_{i}^{*}$ and

$$
x_{2}\left(1-a^{2}\right)=0
$$


for all $a \in A$. We wish to prove that $x_{2}=0$.

Let us suppose that $x_{2} \neq 0$. From (1) we obtain that $A^{2}$ is finite. Let $\widehat{A^{2}}$ denote the sum of all elements of $A^{2}$. If $H$ is a normal subgroup of $G$, then denote by $\Delta(G, H)$ the ideal of $\mathbb{Z} G$ generated by elements of the form $h-1$ with $h \in H$. Clearly,

$$
\mathbb{Z} G / \Delta(G, H) \cong \mathbb{Z}(G / H) .
$$

If $\chi(y)$ is the sum of the coefficients of $y$, then the clement

$$
x+\Delta(G, A)=\chi\left(x_{1}\right)+\chi\left(x_{2}\right) b+\Delta(G, A)
$$

is trivial, because $|G / A|=2[7, \mathrm{p} .46]$. This implies that one of the numbers $\chi\left(x_{1}\right)$ or $\chi\left(x_{2}\right)$ equals \pm 1 and the other is zero. From (1) we obtain $x_{2}=z \widehat{A^{2}}, \quad z \in \mathbb{Z} A, \quad \chi\left(x_{2}\right)=\chi(z)\left|A^{2}\right|$, and this is possible only in the case when $\chi\left(x_{2}\right)=0$.

Suppose $A=A^{2}$. Then $x_{2}=\gamma \sum_{a \in A} a$ for some $\gamma \in \mathbb{Z}$. From the equality $\chi\left(x_{2}\right)=\gamma|A|=0$ we obtain $\gamma=0$ and $x_{2}=0$, which leads to a contradiction. Thus $A \neq A^{2}$. Write $x_{2}=\left(\sum_{i} \alpha_{i} c_{i}\right) \widehat{A^{2}}$ with $\alpha_{i} \in \mathbb{Z}$ where $c_{i}$ 's are a transversal of $A^{2}$ in $A$. Then

$$
\begin{aligned}
x_{1}+x_{2} b+\Delta\left(G, A^{2}\right) & =x_{1}+\left(\sum_{i} \alpha_{i} c_{i}\right) \widehat{A^{2}} b+\Delta\left(G, A^{2}\right) \\
& =x_{1}+\left(\left|A^{2}\right| \sum_{i} \alpha_{i} c_{i}\right) b+\Delta\left(G, A^{2}\right)
\end{aligned}
$$

is a unit in $\mathbb{Z}\left(G / A^{2}\right)$. Since $G / A^{2}$ is an abelian group of exponent two, by Higman's theorem [7, p. 57], all units of $\mathbb{Z}\left(G / A^{2}\right)$ are trivial. Obviously, $\sum_{i} \alpha_{i}=0$ and if $\alpha_{i} \neq 0$ for some $i$, then $\alpha_{i}\left|A^{2}\right| \neq \pm 1$. Thus, $\alpha_{i}=0$ for all $i$ and the equality $x_{2}=0$ is contradictory. Hence, $x=x_{1} \in U(\mathbb{Z} A)$ and $x^{*}=x=x_{1}^{*}=x_{1}$. Clearly, if $x \in U(\mathbb{Z} A)$ and $x^{*}=x$, then $x$ is a central unit of $\mathbb{Z} G$.

It is well known (see [2]) that $U(\mathbb{Z} t(A))= \pm t(A) \times T$ and $U(\mathbb{Z} A)=$ $\pm A \times T$, where every element $u \in T$ satisfies the condition $u=u^{*}$. Therefore the centre of $U(\mathbb{Z} G)$ is the direct product of subgroups $\pm t_{2}(A)$ and $T$. This is 2) of the Proposition.

Suppose that $x=x_{1}+x_{2} b$ is a central unit in $U_{f}(\mathbb{Z} G)$. Since $G$ is a subgroup of $U_{f}(\mathbb{Z} G), \quad x$ is central in $U(\mathbb{Z} G)$. It follows that $x=x_{1}$ and $x x^{f}=x_{1} x_{1}^{*}=x_{1}^{2}= \pm 1$. Therefore, by Higman's theorem $x_{1}= \pm a$ where $a \in t_{2}(A)$. This completes the proof of the Proposition. 
Proof of Theorem 1: Let $G$ be the dikedral of order $2 n$ given by $G=$ $\left\langle a^{n}=1=b^{2}, a^{b}=a^{-1}\right\rangle$. If $n=2$, then the theorem is trivial. So we may apply the last Proposition. Let $Z$ be the centre of $U(\mathbb{Z} G)$. Then we know that $\left(U(\mathbb{Z} G):\left\langle B_{2}(\mathbb{Z} G), Z\right\rangle\right)<\infty$. We have seen in the Proposition above that $Z_{1}$, the centre of $U_{f}(\mathbb{Z} G)$, is finite and $Z_{1}<Z$. It suffices to prove, therefore, that $B_{2}(\mathbb{Z} G)$ is unitary. If $u_{x, y} \neq 0$, then $o(x)=2$ and

$$
u_{x, y}=1+(1-x) y(1+x) .
$$

Now, $y=a^{i} x^{\varepsilon}, \varepsilon=0$ or 1 . Since $x(1+x)=1+x$ we have, in any case,

$$
u_{x, y}=1+(1-x) a^{i}(1+x)
$$

Then $u_{x, y}^{j}=1+(1+x)^{f}\left(a^{i}\right)^{f}(1-x)^{f}=1+(1-x) a^{-i}(1+x)$. Therefore, $u_{x, y} u_{x, y}^{j}=1+(1-x)\left(a^{i}+a^{-i}\right)(1+x)=1$ as $\left(a^{i}+a^{-i}\right)$ is central. This completes the proof of the theorem.

Remark. The last theorem holds for nonabelian groups $G=\langle A, b\rangle$ where $A$ is finitc abelian and $b^{2}=1, a^{b}=a^{-1}$ for all $a \in A$. If $A$ is an elementary 2-group, then so is $G$ and there is nothing to prove. Suppose $A^{2} \neq 1$. The ronlinear irreducible rcpresentations $\rho$ of $G$ are induced from those of $A$ and $\rho(\mathbb{Z} G)=\rho(D)$ for some dihedral subgroup $D$ of $A$. The result follows.

\section{Unitarity of the subgroup $B_{2}(\mathbb{Z} G)$}

Theorem 2. Let $G=\langle A, b\rangle$ where $A$ is the kernel of the nontrivial orientation homomorphism $f: G \rightarrow U(\mathbb{Z})$. The subgroup $B_{2}(\mathbb{Z} G)$ is nontrivial and $f$-unitary if and only if $G$ is non-Hamiltonian in which an element $b \neq 1$ of finite order can be chosen such that one of the following conditions is fulflled:

1) $A$ is an abelian group, the order of the element $b$ divides 4 and $b a b^{-1}=a^{-1}$ for all $a \in A$

2) $A$ is a Hantiltonian 2-group, $G$ is the semidirect product of $A$ and $\left\langle b \mid b^{2}=1\right\rangle$, and every subgroup of $A$ is normal in $G$;

3) $A$ is a Hamiltonian 2-group and $G$ is the direct product of a Hamiltonian 2-subgroup of $A$ and a cyclic group $\langle b\rangle$ of order 4 ;

4) $t(A)$ is an abelion group, every subgroup of $t(A)$ is normal in $G$ and $b a b^{-1}=a^{-1} b^{4 i}$ for all $a \in A$, where the integer $i$ depends on a.

We need the following. 
Lemma. Suppose that $G$ has a subgroup $A$ of index 2 with $G=\langle A, b\rangle$ and $o(b)<\infty$. Suppose further that $A \neq N_{A}(\langle b\rangle)$ and

1) $t(A)$ is abelian and all subgroups of $t(A)$ are normal in $A$;

2) $b g b^{-1}=g^{-1}$ for all $g \in A \backslash N_{A}(\langle b\rangle)$.

Then $b a b^{-1}=a^{-1}$ for all $a \in A$ and $b^{4}=1$.

Proof: Let $c \in N_{A}(\langle b\rangle)$. Choosc $a \in A \backslash N_{A}(\langle b\rangle)$. At first, suppose $c$ has finite order. Then by (2) we have

$$
a^{-1} b c b^{-1}=b(a c) b^{-1}=c^{-1} a^{-1} .
$$

If $a \in t(A)$, then by (1) we have $b c b^{-1}=c^{-1}$. If $a$ has infinite order, there exists an integer $n$ such that $a^{n} c=c a^{n}$, sinces $\langle c\rangle$ is normal in $A$. By hypothesis, $a^{n} c \notin N(\langle b\rangle)$ and thus

$$
a^{-n} b c b^{-1}=b\left(a^{n} c\right) b^{-1}=c^{-1} a^{-n} .
$$

It follows that $b c b^{-1}=c^{-1}$ as desired. Now it is enough to prove that $c$ cannot have infinite order. Suppose that $o(c)=\infty$ and $o(a)<\infty$. Then there is an $n$ such that $e^{n} a=a c^{n}$. Clearly, $a c^{n} \notin N(\langle b\rangle)$. We have

$$
a^{-1} b c^{n} b^{-1}=b\left(a c^{n}\right) b^{-1}=c^{-n} a^{-1} .
$$

It follows that $b c^{n} b^{-1}=c^{-r}$. This is impossible becausc $c^{r_{t}} \in N(\langle b\rangle)$. Now let $o(c)=\infty, \quad o(a)=\infty$. There cxists an $n$ such that $b c^{x_{n}}=c^{n} b$ and $a^{-1} c^{r_{t}}=b a c^{r^{2}} b^{-1}=c^{-r} a^{-1}$. It follows that $\left[c^{n} ; a^{2}\right]=1$. Claarly, $a^{2} c^{n} \notin N(\langle b\rangle)$ and we get.

$$
a^{-2} c^{n}=b a^{2} c^{n} b^{-1}=a^{-2} c^{-n}
$$

which implies $c^{2 n}=1$, a contradiction. Since $b^{2} \in A, b b^{2} b^{-1}=b^{-2}$ and we have $b^{4}=1$, completing the proof of the lemma.

Proof of Theorsm 2:

"Necessity."

Suppose that $B_{2}(\mathbb{Z} G)$ is nontrivial and $f$-unitary. Let us first prove that every finite subgroup $\langle a\rangle$ of $A$ is normal in $G$. Let $n$ be the order of $\langle a\rangle$. If $g \notin N_{G}(\langle a\rangle)$, then $u_{a, g}=1+(1-a) g \bar{a} \neq 1$. Then from the equality $u_{a, g}^{-1}=u_{a, g}^{f}$ we have

$$
\bar{a} g^{-1} f(g)\left(1-a^{-1}\right)=-(1-a) g \bar{a} .
$$

Multiplying by $\bar{a}$ we obtain $n(1-a) g \bar{a}=0$, which is impossible. Therefore, every subgroup of $t(A)$ is norrnal in $G$. Becanse $B_{2}(\mathbb{Z} G) \neq 1, G \backslash A$ 
contains an element $c$ of finite order with $\langle c\rangle$ not normalized by $A$. Then $c^{2} \in t(A)$ and $\overline{c^{2}}$ is central in $\mathbb{Z} G$. Clearly,

$$
u_{c_{1, g}}=1+(1-c) g(1+c) \overline{c^{2}}
$$

and $f(c)=-1$. Since $u_{c, g}$ is $f$-unitary, $u_{c, g} u_{c, g}^{f}=1$ and it follows that

$$
\left(g+g^{-1} f(g)\right)(1+c) \overline{c^{2}}=c\left(g+g^{-1} f(g)\right)(1+c) \overline{c^{2}} .
$$

Choose $b \in G \backslash A$ such that $b$ is a 2-elcment of least order and let $g \in A$. In (1) taking $c=b, g=b g^{-1} b^{1+2 i}$ whencver $g \notin N_{A}(\langle b\rangle)$. We obtain $b g b^{-1}=g^{-1} b^{2 i^{\prime}}$ for all $g \in A \backslash N_{A}(\langle b\rangle)$ and

$$
(b g)^{2}=\left(g^{-1} b^{2} g\right)^{i^{\prime}+1} .
$$

Clearly, $b g$ is a 2-element in $G \backslash A$ and $i^{\prime}$ is even, otherwise the order of $b g$ is less than the order of $b$, which is impossible. Therefore,

$$
b g b^{-1}=g^{-1} b^{4 j}
$$

for all $g \in A \backslash N_{A}(\langle b\rangle)$.

a) Suppose that the order of $b$ divides 4 .

Then from (2) $b g b^{-1}=g^{-1}$ for all $g \in A \backslash N_{A}(\langle b\rangle)$.

If $t(A)$ is abclian, then, by the Lemma, $A$ is abelian and $b a b^{-1}=a^{-1}$ for all $a \in A$. This is case 1) of the theorem.

If $t(A)$ is nonabelian, then $t(A)$ is a Hamiltonian group and

$$
t(A)=Q \times E \times T
$$

wherc $Q$ is the quaternion group of order $8, E^{2}=1$ and all elements of $T$ are of odd order.

We wish to prove that $A=t(A)$. Suppose that $g$ is an element of infinite order of $A \backslash N(\langle b\rangle)$. Then $g^{2} \in C_{A}(Q)$ and there exists an element $w$ of order 4 of $Q$ such that $[b, w]=1$, because every subgroup of $Q$ is normal in $G$. Clearly, $g^{2} w \notin N(\langle b\rangle)$ and by (2)

$$
w g^{-2}=b w g^{2} b^{-1}=b g^{2} w b^{-1}=w^{-1} g^{-2},
$$

which is impossible. Therefore, all elements of $A \backslash N(\langle b\rangle)$ have finite orders.

Let $g$ be an element of infinite order from $N_{A}(\langle b\rangle)$ and let an $a \in$ $A \backslash N_{A}(\langle b\rangle)$. Clearly there exists $n$ such that $\left[g^{n}, a\right]=1$, because the finite 
cyclic subgroup $\langle a\rangle$ is norrmal in $G$. Then $g^{n} a \in A \backslash N_{A}(\langle b\rangle)$ and $g^{n} a$ is of infinitc ordcr, which leads to a contradiction. Therefore, $t(A)=A$.

We claim that $T=1$. Let $v$ be an element of odd order from $A \backslash N(\langle b\rangle)$. Obviously, there exists an clement $w$ of order 4 in $Q$ such that $[b, w]=1$, as every subgroup of $Q$ is normal in $G$. Thus $v w \notin N(\langle b\rangle)$ and by (2)

$$
v^{-1} w=b v w b^{-1}=w^{-1} v^{-1},
$$

which is impossible. Next, let $v$ be an element of odd order from $N_{A}(\langle b\rangle)$. Because $\langle v\rangle \triangleleft G,[v, b]=1$. Clearly there is an element $w$ of order 4 in $Q$ such that $b^{-1} w b=w^{-1}$ and $v w \notin N_{A}(\langle b\rangle)$. Then

$$
w^{-1} v=b w v b^{-1}=v^{-1} w^{-1},
$$

which is impossible. Hencc, the structure of $G$ is described in case 2) or 3) of the theorem.

b) Suppose that the order of $b$ is $2^{k} \quad(k \geq 3)$.

Then by (2) $b^{2}$ belongs to the centre of $t(A)$, because $t(A)$ is abelian or Hamiltonian. Hence, $t(A)$ is abelian and every subgroup of $t(A)$ is normal in $G$. Then from (2) $b a b^{-1}=a^{-1} b^{4 j}$ for all $a \in A \backslash N_{A}(\langle b\rangle)$. Denote by $\left\langle b^{4 r}\right\rangle$ the subgroup generated by $b^{1 j}=a b a b^{-1}$, as a runs over $A \backslash N_{A}(\langle b\rangle)$.

Put $\widetilde{G}=G /\left\langle b^{4 r}\right\rangle, \quad \widetilde{A}=A /\left\langle b^{4 r}\right\rangle$ and $\widetilde{b}=b\left\langle b^{4 r}\right\rangle$. Then $\widetilde{G}$ satisfies the conditions of our Lernma and it follows that $r=1$ and $\widetilde{b} a \widetilde{b}^{-1}=a^{-1}$ for all $a \in \widetilde{A}$. This is case 4) of the theorem.

"Sufficiency."

Let $G$ satisfy one of the conditions 1)-4) of the theorem. Clearly, if a finite subgroup $\langle c\rangle$ is not normal in $G$, then $c \in b A,\left\langle c^{2}\right\rangle=\left\langle b^{2}\right\rangle$ and $\overline{c^{2}}$ belongs to the centre of $\mathbb{Z} G$. Therefore,

$$
u_{c, 3}=1+(1-c) g(1+c) \overline{c^{2}}
$$

and

$$
u_{c_{, g} g} u_{c, g}^{f}=1+(1-c)\left(g+g^{-1} f(g)\right)(1+c) \overline{c^{2}} .
$$

Suppose that $g \in A$. Then $f(g)=1$ and $\left(g+g^{-1}\right) \overline{c^{2}}$ is a central element. This is obvious in cases 1), 2) and 3). Suppose that $G$ satisfies the condition 4) of the theorem. Then $\left\langle c^{2}\right\rangle=\left\langle b^{2}\right\rangle$, and $b g b^{-1}=g^{-1} b^{4 i}$ and $G /\left\langle b^{4}\right\rangle$ is abelian. Thus

$$
b\left(g+g^{-1}\right) \overline{c^{2}} b^{-1}=\left(g+g^{-1}\right) \overline{c^{2}}=a^{-1}\left(g+g^{-1}\right) \overline{c^{2}} a
$$

and $\left(g+g^{-1}\right) \overline{c^{2}}$ is central in $\mathbb{Z} G$. 
If $g \in b A$, then $g=b a, \quad f(g)=-1$ and

$$
g^{-1}=a^{-1} b^{-1}=b^{-1} a b^{4 i} .
$$

Clearly, $g^{-1} \overline{c^{2}}=g \bar{c}^{2}$ and $\left(g+f(g) g^{-1}\right) \overline{c^{2}}=0$. Therefore, $u_{c, g} u_{c, g}^{f}=$ 1 and the bicyclic units are $f$-unitary. Thus $B_{2}(\mathbb{Z} G)$ is an $f$-unitary subgroup, proving the theorem

\section{References}

1. A.A. BovDl, Unitary subgroup of the multiplicative group of integral group ring of a cyclic group, Math. Zametki 41 (4) (1987), $467-474$.

2. A.A. BovDl, The multiplicative group of an integral group ring, Uzhgorod, 1987.

3. K. Holeuismann and S.K. Sehgal, On a thcorem of Bovdi, to appear.

4. S.P. Novikov, Algebraic construction and properties of Hermitian analogues of $K$-theory over rings with involution from the viewpoint of Hamiltonian formalism, Applications to differential topology and the theory of characteristic classes, II, Izv. Akad. Nauk SSSR Ser. Mat. 34 (1970), 475-500; English transl. in Math. USSR Izv. 4 (1970).

5. J. RitTlir AND S.K. SEItgat, Generators of subgroups of $U(\mathbb{Z} G)^{*}$, Contemporary Math. 93 (1989), $331 \cdot 347$.

6. J. Rin'Ter AND S.K. SEngni, Construction of units in integral group rings, Trans. A.M.S. 324 (1991), 603-621.

7. S.K. Skilcsl, "Topics in group rings," M. Dekker, New York, 1978.

A.A. Bovdi:

Mathematics Irstitute

Uahgorod State University

U\%ligorod

USSIR
S.K. Sehgal:

Department of Matherratics

University of Alberta

Edrnoriton, Alberta

CANADA TGG 2G1 